\title{
Relation entre la diversité du substrat et la diversité faunistique dans un bief belge de la rivière Meuse
}

\author{
M. Evrard1 \\ J.-C. Micha1
}

Mots clés : microhabitat, substrat, aménagements, Meuse, macrobenthos, rivière, Waulsort.

Une étude quantitative des peuplements d'invertébrés benthiques en relation avec certains substrats naturels et artificiels, utilisés pour la consolidation des berges, a été réalisée dans 9 stations du bief de Waulsort lors de la mise en chômage technique de la Meuse (Belgique). Les stations à grande hétérogénéité physique sont caractérisées par une richesse et une diversité faunistique importante ( 33 familles, 15790 ind. $/ \mathrm{m}^{2}, \mathrm{H}^{\prime}=2,08$ ) de même qu'une colonisation optimale de substrat. Les stations à substrats artificiels, fortement homogènes, restent peu accueillantes pour la faune benthique (17 familles, 1352 ind. $/ \mathrm{m}^{2}, \mathrm{H}^{\prime}=0,52$ ). Dans la perspective de l'aménagement futur des berges du barrage de Waulsort, la construction de perrés non cimentés à faible pente doit être privilégiée.

\section{Relation between the substrate diversity and the faunistic diversity in a reach of the river Meuse (Belgium)}

Keywords : microhabitat, substrate, managements, Meuse, macrobenthos, river, Waulsort.

A quantitative study of the benthic invertebrates communities in relation with some natural and artificial substrate, used for the banks reinforcing, has been carried out in 9 sites of the Waulsort reach during the technical shutting down of the river Meuse (Belgium). The sites with high physical heterogencity are caracterised by a high faunistic richness, a high diversity (33 famillies, 15790 ind. $/ \mathrm{m}^{2}, \mathrm{H}^{\prime}=2,08$ ) and by a optimal colonisation of the substrate. The sites with artificial substrate, heavily homogeneous, are weakly welcoming for the benthic fauna (17 famillies, $1352 \mathrm{ind} / \mathrm{m}^{2}$, $H^{\prime}=0,52$ ). In the view of the coming arrangement of the banks of the Waulsort sluice, the building of a shelved wall with non-cemented bricks must be priviliged.

\section{Introduction}

La Meuse, le plus important des cours d'eau belge, avec ses 925 kilomètres, dont 124 en Belgique, prend sa source en France au pied du plateau de Langres à $\mathbf{4 1 0}$ mètres d'altitude et se jette dans la Mer du Nord après avoir traversé les Pays-Bas, où elle se divise en de nombreux bras pour former un estuaire commun avec le Rhin (Fig. 1).

Le gabarit actuel de la Meuse pour la navigation en amont de Namur est de 1350 tonnes alors qu'en aval le gabarit de 9000 tonnes est atteint depuis 1989 (Micha \& Borlée 1989). Les impératifs de la navigation ont nécessité la modernisation des barrages, la consolidation des berges par des perrés obliques

1. Unité d'Ecologie des Eaux Douces (UNECED), Facultés Universitaires Notre-Dame de la Paix, rue de Bruxelles, 61, B-5000 Namur, Belgium. ou par des murs bétonnés (Verniers et al. 1991). De ce fait, l'entretien des barrages et des écluses répartis le long de la Meuse, provoque la mise en chômage technique du cours d'eau. Cette opération qui consiste à ouvrir les barrages, a pour conséquence une importante baisse du niveau de l'eau. Les conditions exceptionnelles offertes pendant cette période permettent d'effectuer des échantillonnages quantitatifs tout le long du profil exondé de la berge.

\section{Description du site}

L'impact des aménagements de la rivière se traduit par une différence fondamentale entre la rive droite et la rive gauche de la Meuse (Micha \& Pilette 1988, Micha \& Borlée 1989). Dans le bief de Waulsort que nous avons plus particulièrement étudié, près de $40 \%$ des berges en rive droite sont naturelles (Fig. 1). En rive gauche, la berge est surélevée et artificielle à $100 \%$ car elle supporte la route 
nationale et la voie de chemin de fer Namur-Dinant. Le talus est soit un simple mur bétonné, soit un perré à pierres cimentées, recouvert par endroits d'une coulée de béton.

Depuis quelque temps, l'Unité d'Ecologie des Eaux Douces (UNECED) des Facultés Universitaires Notre-Dame de la Paix de Namur (Belgique) a entrepris une étude globale de la Meuse en tant qu'écosystème fluvial. Décrire et préciser le préférendum des espèces qui font partie intégrante de cet écosystème s'inscrit donc dans le cadre général de cette étude de la Meuse. L'étude des substrats présents dans le bief de Waulsort et la microdistribution qu'ils imposent aux macroinvertébrés benthiques permet de rendre compte de la biodiversité d'un fleuve en fonction de la mosaïque de substrats.

L'objectif de ce travail est de décrire et de comparer différentes communautés macrobenthiques réparties sur différents substrats artificiels et sur certains substrats naturels dans un bief (Waulsort) relativement épargné par les aménagements successifs des rives de la Meuse. L'organisation de cette campagne d'échantillonnage fut également motivée par l'absence de données quantitatives de ce type dans une zone du potamon profond de la Meuse, difficilement accessible en temps normal.

\section{Matériel et méthodes}

Afin de préciser la relation entre la diversité benthique et la structure du substrat, il était nécessaire de choisir des stations de substrats distincts afin de les comparer au point de vue de leur texture mais surtout de leur affinité pour un type de faune benthique. L'intérêt du bief de Waulsort est l'existence de nombreux sites naturels à faciès lentique où le courant permet une sédimentation des éléments fins transportés, mais également des sites à faciès typiquement lotique dans lesquels aucun dépôt n'est possible, le fond étant régulièrement érodé. Les autres biefs situés entre Namur et Waulsort sont largement aménagés.

Le choix des stations s'est donc effectué à partir de la carte d'aménagement des berges de la Meuse moyenne supérieure (Verniers 1990). Notre choix s'est porté d'abord sur les berges à substrats artificiels c'est-à-dire mis en place par l'homme ; c'est ainsi que les perrés cimentés ou non, avec ou sans coulée de béton ont été sélectionnés (Fig. 1). Quant aux berges naturelles, différents substrats ont été choisis en fonction de leur texture, celle-ci variant des plages de galets aux plages vaseuses en passant par les berges à caractères plus caillouteux. Dans notre étude, le terme caillouteux comprend des éléments minéraux variant de 5 à 25 centimètres répartis de façon aléatoire sur la berge. Les stations ont également été réparties le long du bief de Waulsort afin de rendre compte de l'évolution de la vitesse du courant dans le bief en fonction de l'éloignement par rapport au barrage de Waulsort.

Nous avons profité de la mise en chômage technique de la Meuse le 16 septembre 1989 pour réaliser en deux jours (les 17 et 18 septembre 1989) nos prélèvements de macroinvertébrés dès le début du chômage technique. Afin d'obtenir une représentativité optimale de la faune benthique, neuf échantillons, disposés le long du profil exondé de la berge, ont été prélevés dans chacune des 9 stations, soit au total 81 échantillons (Fig. 2). Trois premiers échantillons ont été effectués à l'aide de quadrats dans une zone $\mathrm{A}$ comprise entre le haut de berge et 0,5 mètres en contre-bas de celui-ci. Trois autres échantillons (zone B) ont été prélevés avec des quadrats entre 0,5 et 2 mètres du haut de berge, enfin la dernière série de trois échantillons (zone C), prélevés à l'aide d'un filet Surber, s'étend à plus de 2 mètres à partir du haut de berge et est généralement située au-delà du niveau d'étiage. Cette subdivision des berges, réalisée en respect du profil transversal de la berge, permet l'échantillonnage de l'ensemble des substrats et aussi de rendre compte d'une représentativité faunistique suffisante. Les deux moyens de prélèvement ont été ramenés à une surface identique afin de permettre des comparaisons quantitatives. Le filet de Surber de $0,1 \mathrm{~m}^{2}$ (vide de maille de $0,3 \mathrm{~mm}$ ) est disposé au hasard sur le substrat immergé. Pour l'échantillonnage à l'aide de quadrats, le cadre de $0,1 \mathrm{~m}^{2}$ est lancé au hasard sur le substrat exondé. L'échantillon est raclé sur une profondeur de quelques centimètres à l'aide d'une truelle et est déversé dans un sac plastique contenant du formol $5 \%$. Tous les échantillons prélevés par quadrats, sont utilisés dans la subdivision supérieure (zone A) et intermédiaire (zone $B$ ) de la berge, le premier jour de la campagne d'échantillonnage (Fig. 2). Les échantillons prélevés avec le filet Surber et se situant dans la subdivision inférieure (zone $\mathrm{C}$ ) ont été récoltés le second jour. 


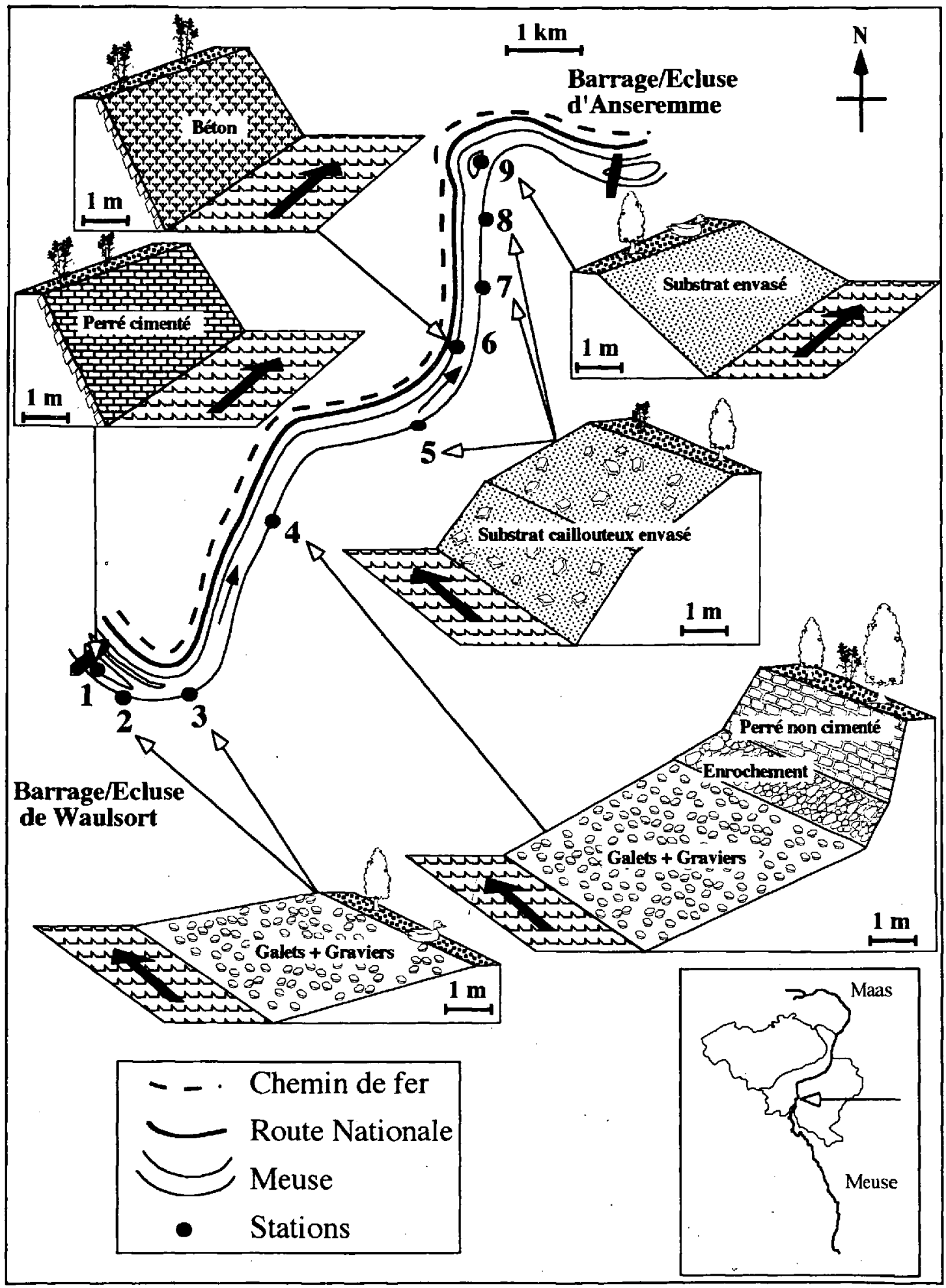

Fig. 1. Localisation des stations et caractéristiques des substrats exondés du bief de Waulsort lors de la mise en chômage technique de la Meuse en Belgique (septembre 1989), le terme « caillouteux » regroupe les éléments minéraux de 5 à 25 centimètres de diamètre.

Fig. 1. Location of the sampling sites and characteristics of the substrates in the Waulsort reach during the technical shutting down of the river Meuse in Belgium (September 1989). 


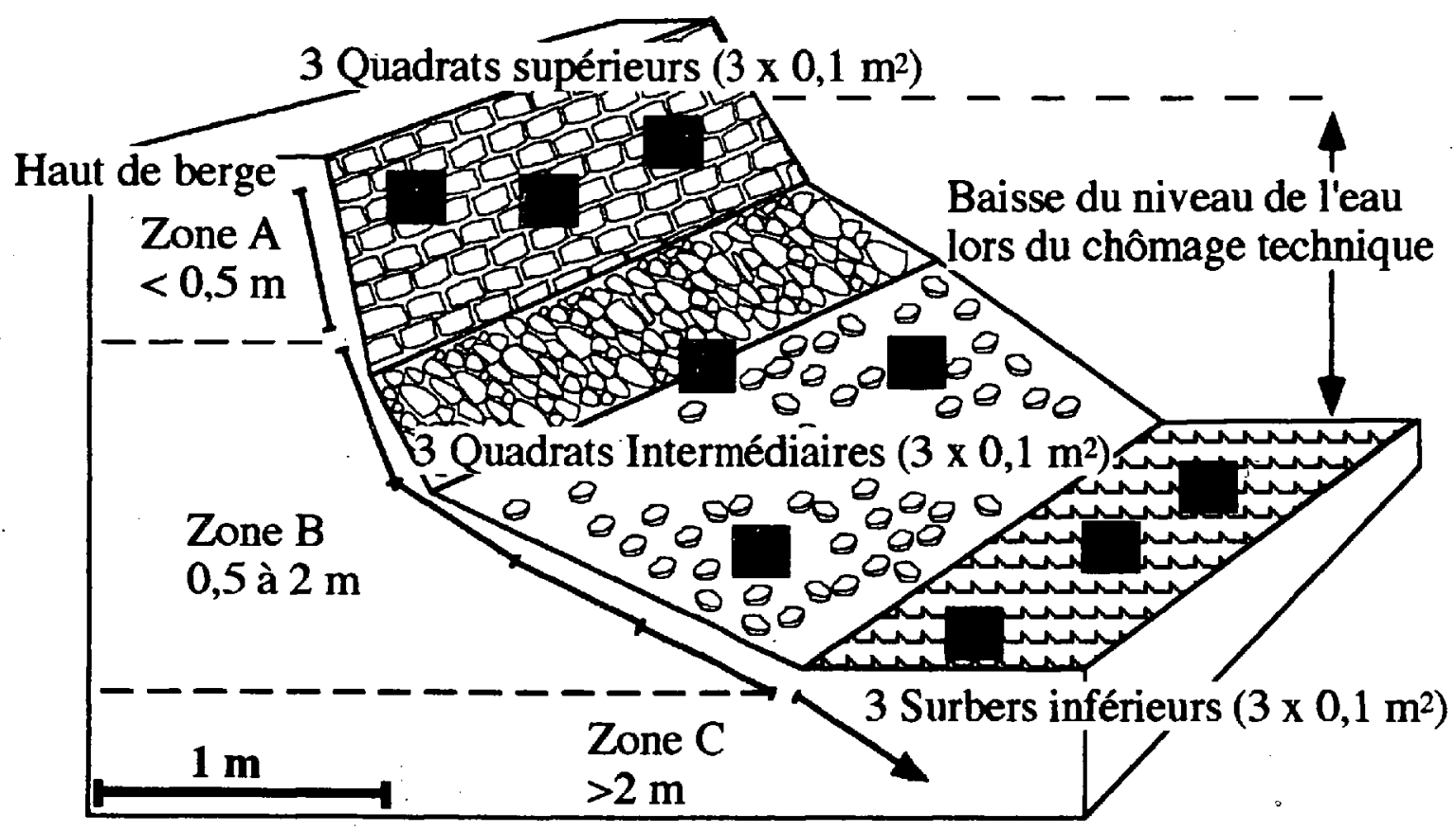

Fig. 2. Disposition des 6 quadrats et 3 filets Surber dans les trois zones d'échantillonnage du substrat d'une berge exondée (Waulsort, septembre 1989).

Fig. 2. Arrangement of the 6 quadrats and the 3 Surber nets on three sampling zones of the substrate on emerged banks (Waulsort, September 1989).

Afin de rendre compte de la répartition aléatoire des individus, nous avons choisi de calculer l'indice de diversité d'après la méthode de Shannon-Weaver (Dajoz 1971). 11 s'exprime suivant : $\mathrm{H}^{\prime}=-\Sigma\left(\mathrm{n}_{\mathrm{i}} / \mathrm{N}\right)$ $\ln \left(n_{\mathrm{i}} / \mathrm{N}\right) ;$ où $\mathrm{H}^{\prime}=$ indice de diversité $; \mathrm{n}_{\mathrm{i}}=$ nombre d'individus du taxon $\mathrm{i} ; \mathrm{N}=$ nombre total d'individus. Cet indice reflète les modifications de la structure des peuplements et visualise leurs variations dans l'espace. Plus H' est élevé, plus le milieu contient un nombre d'espèces à abondance relative élevée, impliquant une communauté benthique diversifiée.

\section{Résultats}

\subsection{Diversité du substrat}

Neuf types de substrats ont été examinés en fonction de leur structure (Fig. 3), artificielle ou non, et en fonction de leur granulométrie : il s'agit de substrats composés de galets et de graviers par opposition aux substrats exclusivement envasés. Les substrats caillouteux envasés présentent un substrat recouvert d'une couche de vase de quelques millimètres.

La surface exondée des substrats naturels $(60,5 \%)$ du bief de Waulsort dépasse celle des substrats artificiels $(39,5 \%)$. Les substrats naturels envasés ne constituent que $8,8 \%$ de la superficie totale exondée, quatre types de substrats artificiels occupent chacun des proportions similaires ( 9 aे $10 \%$ ). Les gabions et le mur vertical restent faiblement représentés. De toute évidence, les substrats naturels composés de galets et de graviers dominent en compagnie des substrats naturels caillouteux envasés avec respectivement $23,5 \%$ et $28,2 \%$.

En ce qui concerne les vitesses du courant mesurées en surface quelques jours avant la mise en chômage technique de la Meuse, seules les stations 2, 3 et 4 montrent des vitesses du courant élevées comprises entre 50 et $80 \mathrm{~cm} / \mathrm{s}$. Les stations restantes possèdent toutes des vitesses du courant inférieures à $10 \mathrm{~cm} / \mathrm{s}$, voire nulles, y compris la station 1 pourtant 


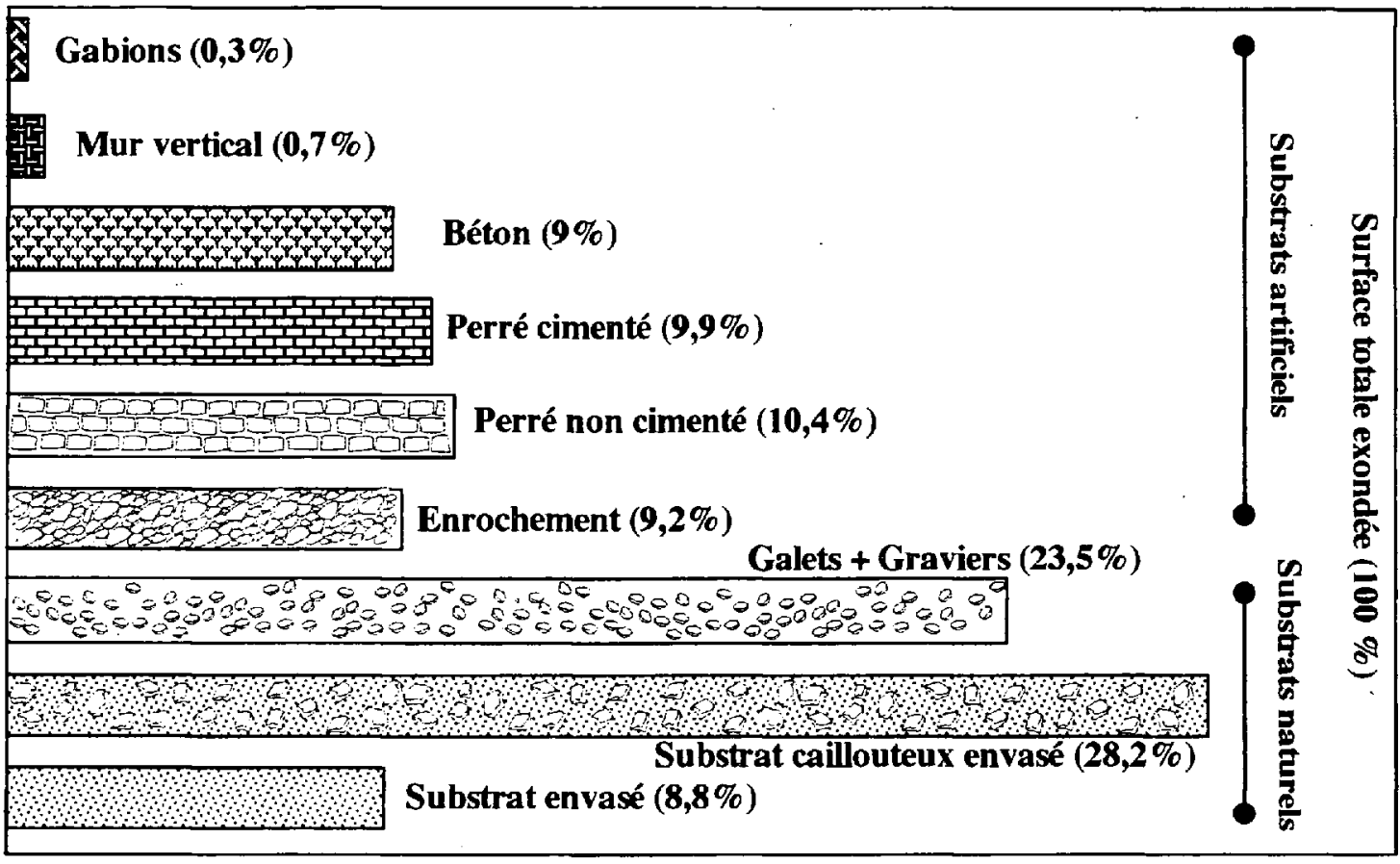

Fig. 3. Répartition des différents types de substrats naturels et artificiels exondés dans l'ensemble du bief de Waulsort.

Fig. 3. Distribution of natural and artificial emerged substrates in the Waulsort reach (September 1989).

localisée au pied du barrage de Waulsort mais située en retrait dans une zone de remous. Cette variation considérable de la vitesse du courant le long du bief de Waulsort visualise l'effet des barrages mosans sur la masse d'eau retenue et influence directement l'aspect du substrat dans les différentes stations, principalement celles présentant des plages de galets et des gravières proches du barrage.

Les 81 échantillons, regroupés au sein de chacune des 9 stations (Tableau 1), comptent près de 60000 individus répartis dans 38 familles distinctes, accompagnées de quelques Nématodes dans certaines stations.

Les deux types de berge présentant la plus forte colonisation en nombre d'individus sont les stations à substrat nettement pierreux (galets et graviers) : la station 2 avec 14211 individus (15 $790 \mathrm{ind} . / \mathrm{m}^{2}$ ) et la station 3 avec 9586 individus (10 651 ind. $/ \mathrm{m}^{2}$ ) (Fig. 4). Les valeurs obtenues pour les stations 4 , 7 et 9 sont proches l'une de l'autre avec un nombre d'individus variant de 7000 a 8000 individus celles des stations 1,5 et 8 comptent moins de 4500 individus par station. Le résultat exceptionnel du perré cimenté (station 1) doit être nuancé. En effet Corophium curvispinum représente près de $90 \%$ des individus. Si l'on ne considère pas cette espèce, la station apparaît comme l'une des moins peuplées. Une seule station est vraiment très peu colonisée, celle du perré recouvert d'une coulée de béton (station 6) où l'ensemble des 9 prélèvements ne contenait que 1217 individus ( 1352 ind. $/ \mathrm{m}^{2}$ ).

L'analyse globale des stations (Fig. 4) révèle que l'indice de diversité de Shannon-Weaver le plus faible $\left(\mathrm{H}^{\prime}=0,52\right)$ est celui du perré cimenté (station 1). Il est supérieur mais toujours compris entre 1 et 2 dans l'ensemble des autres stations. Seul le perré non cimenté de la station 4 avec sa plage de galet en pente douce se révèle intéressant du point de vue diversité faunistique avec l'indice le plus élevé $\left(\mathrm{H}^{\prime}=2,08\right)$.

Cette valeur élevée de l'indice reflète la forte hétérogénéité du substrat à la station 4. En effet, le substrat correspondant est plus diversifié qu'aux stations 


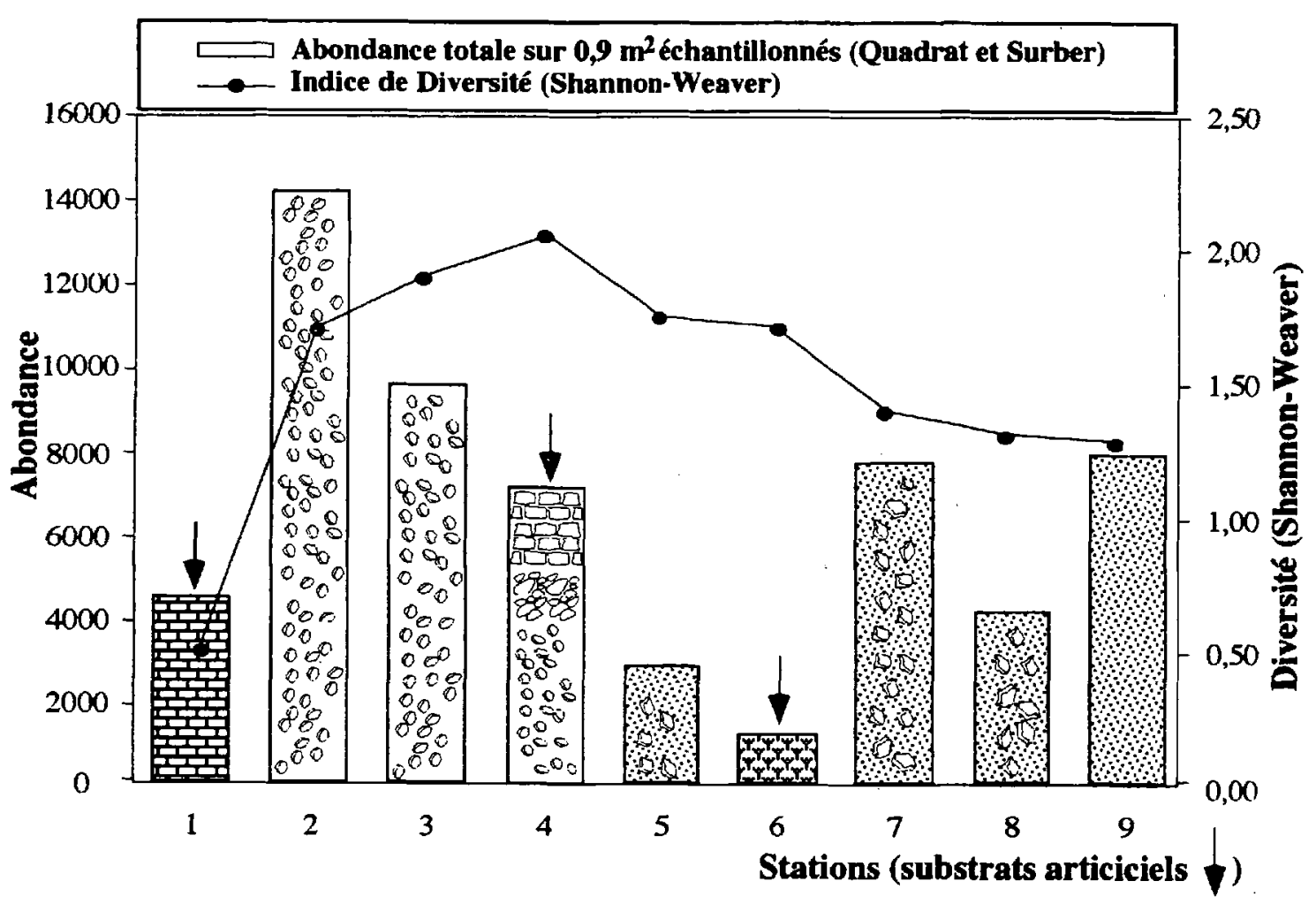

Fig. 4. Evolution des effectifs et de l'indice de diversité de Shannon-Weaver sur une surface échantillonnée de $0.9 \mathrm{~m}^{2}$ dans chacune des 9 stations du bief de Waulsort (septembre 1989).

Fig. 4. Evolution of the number of inviduals and evolution of the Shannon-Weaver index on a $0.9 \mathrm{~m}^{2}$ sampled surface in 9 stations of the Waulsort reach (September 1989).

2 et 3 qui pourtant hébergent un nombre d'individus et de taxa plus élevés. En plus des stations 2 et 3 qui ne possèdent que des galets, des graviers pour cette dernière, la station 4 présente non seulement une plage de galets avec du gravier, mais aussi, un perré non cimenté avec un enrochement artificiel de blocs à sa base. Ces structures hétérogènes représentent, par excellence, une diversité de microhabitats très appréciée par les macroinvertébrés benthiques qui y trouvent refuge et nourriture. Tout a fait à l'opposé de ce substrat hétérogène, nous trouvons le substrat homogène type c'est-à-dire le perré cimenté de la station 1 qui ne permet guère la colonisation par les macroinvertébrés. Les substrats envasés se maintiennent entre ces deux extrêmes, avec une hétérogénéité faunistique d'autant plus grande qu'ils comportent une composante " caillouteuse ".
Que ce soit à un niveau de détermination plus poussé (genre ou espèce) ou au niveau des familles, l'analyse de la richesse taxonomique confirme les observations précédentes (Fig. 5). Les stations présentant la plus grande richesse taxonomique sont encore une fois celles aux plages de galets et de graviers : stations 2 et 3 avec respectivement 31 et 33 familles. L'ancien perré de la station 4 avec un substrat de même type, possède une faune comprenant 24 familles. La station 6 , celle du perré à coulée de béton, est de nouveau la moins variée avec 17 familles. Le perré cimenté de la station 1 présente une richesse taxonomique limitée, toutefois le léger envasement qui subsiste à sa base favorise une richesse taxonomique légèrement supérieure à celle de la station 6 . Les stations restantes correspondent toutes à un faciès lentique et comprennent un substrat principalement vaseux, constitué de sédiments (stations 5, 7,8 et 9). 
Tableau 1. Densité (ind. $/ 0.9 \mathrm{~m}^{2}$ ) dans chacune des 9 stations échantillonnées en septembre 1989 dans le bief de Waulsort lors du chômage technique de la Meuse en Belgique. Certains genres et espèces sont regroupés par familles.

Table 1. Density (ind. $/ 0.9 \mathrm{~m}^{2}$ ) in the Waulsort reach during the technical shutting down of the river Meuse in Belgium (September 1989). Some genera and species are grouped within families.

\begin{tabular}{|c|c|c|c|c|c|c|c|c|c|c|}
\hline Taxa & Stations & 1 & 2 & 3 & 4 & 5 & 6 & 7 & 8 & 9 \\
\hline Nématodes & & 1 & 1 & 3 & 2 & & & 1 & & 1 \\
\hline \multirow[t]{4}{*}{ Triclades } & Dendrocoelun lacteum (Mül.) & & 3 & 1 & 4 & & & & & \\
\hline & Dugesia tigrina (Gir.) & & 2 & 2 & & & & & & \\
\hline & Dugesia lugubris Schm. & 70 & 2114 & 649 & 321 & 108 & 17 & 80 & 4 & 2 \\
\hline & Polycelis sp. & & & & 1 & & & & & \\
\hline \multirow[t]{6}{*}{ Lamellibranches } & Dreissena polymorpha Pallas & & 3 & 1 & & & & & & \\
\hline & Pisidium spp. & & 39 & 161 & 37 & 359 & & 258 & 378 & 157 \\
\hline & Sphaeriuan spp. & 6 & 162 & 168 & 109 & 5 & 10 & 4 & & 13 \\
\hline & Anodonta anatina $\mathrm{L}$ & & & 9 & 1 & 2 & & & 1 & 5 \\
\hline & Unio pictorum $\mathrm{L}$ & & & 4 & 2 & 16 & & 7 & 4 & 10 \\
\hline & Unio crassus Phlps. & & ' & & 1 & 1 & & & & 1 \\
\hline \multirow[t]{6}{*}{ Gastéropodes } & Ancylus fuviatilis (Mull.) & & 3 & & & & & & & \\
\hline & Bithynia tentaculata (L.) & 1 & 7 & 21 & 12 & 1 & 9 & 8 & 1 & 6 \\
\hline & Lymnaea spp. & & & 11 & & & 5 & 26 & 13 & 4 \\
\hline & Theodoxus funianilis (L.) & 1 & 47 & 5 & & 3 & & & & 5 \\
\hline & Valvata piscinalis (Mull.) & & 1 & 1 & & 14 & & 9 & 12 & $\mathbf{1}$ \\
\hline & Viviparus viviparus L. & & & 1 & & 5 & 3 & & & 1 \\
\hline \multirow[t]{4}{*}{ Oligochètes } & Lumbricidae & & 3 & 58 & 124 & 1 & & 1 & 3 & 1 \\
\hline & Lumbriculidae & 28 & 780 & 1707 & 762 & 897 & 29 & 400 & 495 & 3587 \\
\hline & Tubificidae & 3 & 128 & 237 & 192 & 36 & 1 & 31 & 7 & 269 \\
\hline & Branchiwra sowerbyi Bedd. & & 61 & 16 & 110 & 22 & & 3 & 18 & 20 \\
\hline \multirow[t]{4}{*}{ Achètes } & Erpobutella octoculata (L) & 6 & 38 & 15 & 13 & & & & & \\
\hline & Glossiphonia complanata (L) & 2 & 6 & 5 & 14 & & & 2 & 3 & 1 \\
\hline & Helobdella stagnalis (L) & & & 30 & 24 & & 8 & 1 & 8 & 20 \\
\hline & Piscicola geomelra (L) & & & 2 & & 3 & & 3 & & \\
\hline \multirow[t]{7}{*}{ Crustacés } & Asellus aquaticus $\mathrm{L}$ & 6 & 60 & 108 & 456 & 105 & 39 & 28 & 6 & 3 \\
\hline & Proasellus meridianus Racovitza & & 7 & 10 & 13 & • & & & & \\
\hline & Corophium curvispinum Sars & 4071 & 2736 & 3461 & 2216 & 25 & 159 & 158 & 8 & 31 \\
\hline & Gammanus pulex (L.) & 5 & 89 & 85 & 114 & 31 & 7 & 134 & 5 & 38 \\
\hline & Gammarus roeselii (Gervais) & & & & & & & 1 & I & 1 \\
\hline & Echinogammarus berilloni (Catta) & & 18 & 5 & 6 & 1 & & 1 & 1 & 1 \\
\hline & Orconectes limosus Raffinesque & & $\mathbf{J}$ & 2 & 4 & 1 & & & & \\
\hline \multirow[t]{4}{*}{ Ephéméroptères } & Baetis spp. & & & 4 & 1 & & & & & \\
\hline & Caenis spp. & 19 & 74 & 29 & 23 & 3 & 2 & 9 & 2 & \\
\hline & Ephemera danica Mlill. & & 9 & 1 & 2 & & 2 & $\therefore$ & 1 & \\
\hline & Ecdyonurus sp. & 1 & 1 & & & & . & & & \\
\hline \multirow[t]{3}{*}{ Odonates } & Calopteryx virgo (L.) & & 4 & & & & . & $i$ & & \\
\hline & Gomphus vulgatissimus (L.) & & & & & 5 & & & & \\
\hline & Platycnemis pennipes (Pallas) & 3 & 54 & 3 & 1 & & & & 1 & . \\
\hline \multirow[t]{3}{*}{ Coléoptères } & Dystiscidae & & & 1 & & & & 7 & & 2 \\
\hline & Elmis spp. & $\overrightarrow{1}$ & & & & & . & & & \\
\hline & Limnius spp. & 2 & 3 & 1 & 1 & & 1 & 2 & & 2 \\
\hline Hétéroptères & Aphelocheinus aestivalis Fab. & 8 & 1 & & & & & 1 & & \\
\hline Mégaloptères & Sialis nigripes Pict. & & & 10 & 11 & & & 2 & & \\
\hline \multirow[t]{7}{*}{ Trichoptères } & Ceraclea spp. & & 9 & 5 & & & & $\cdot$ & & \\
\hline & Mystacides spp. & & 5 & 2 & & 1 & & 3 & & \\
\hline & Cyrnus trimaculans Curt. & & 3 & 17 & 7 & 2 & 12 & & 1 & \\
\hline & Hydropsyche contubernalis $\mathrm{McL}$. & 55 & 48 & & & & & & & \\
\hline & Hydropsyche exocellata Duff. & 2 & 3 & & & & & & & \\
\hline & Neureclipsis bimaculata $\mathrm{L}$ & & 18 & & 1 & & & & & \\
\hline & Ecnomus tenellus Ramb. & 10 & 543 & 187 & 121 & 2 & 13 & 32 & 5 & \\
\hline \multirow[t]{4}{*}{ Diptères } & Atherix ibis (F.) & 3 & 2 & & & & & & & \\
\hline & Chironomidae & 36 & 482 & 378 & 313 & 109 & 128 & 112 & 120 & 229 \\
\hline & Glyptotendipes spp. & 135 & 6643 & 2155 & 2053 & 1081 & 570 & 3281 & 572 & 301 \\
\hline & Chironomus spp. & & & 15 & 87 & 55 & 202 & 3192 & 2478 & 3165 \\
\hline \multicolumn{2}{|c|}{ Richesse taxunomique globale } & 24 & 40 & 42 & 35 & 28 & 19 & 30 & 26 & 28 \\
\hline
\end{tabular}



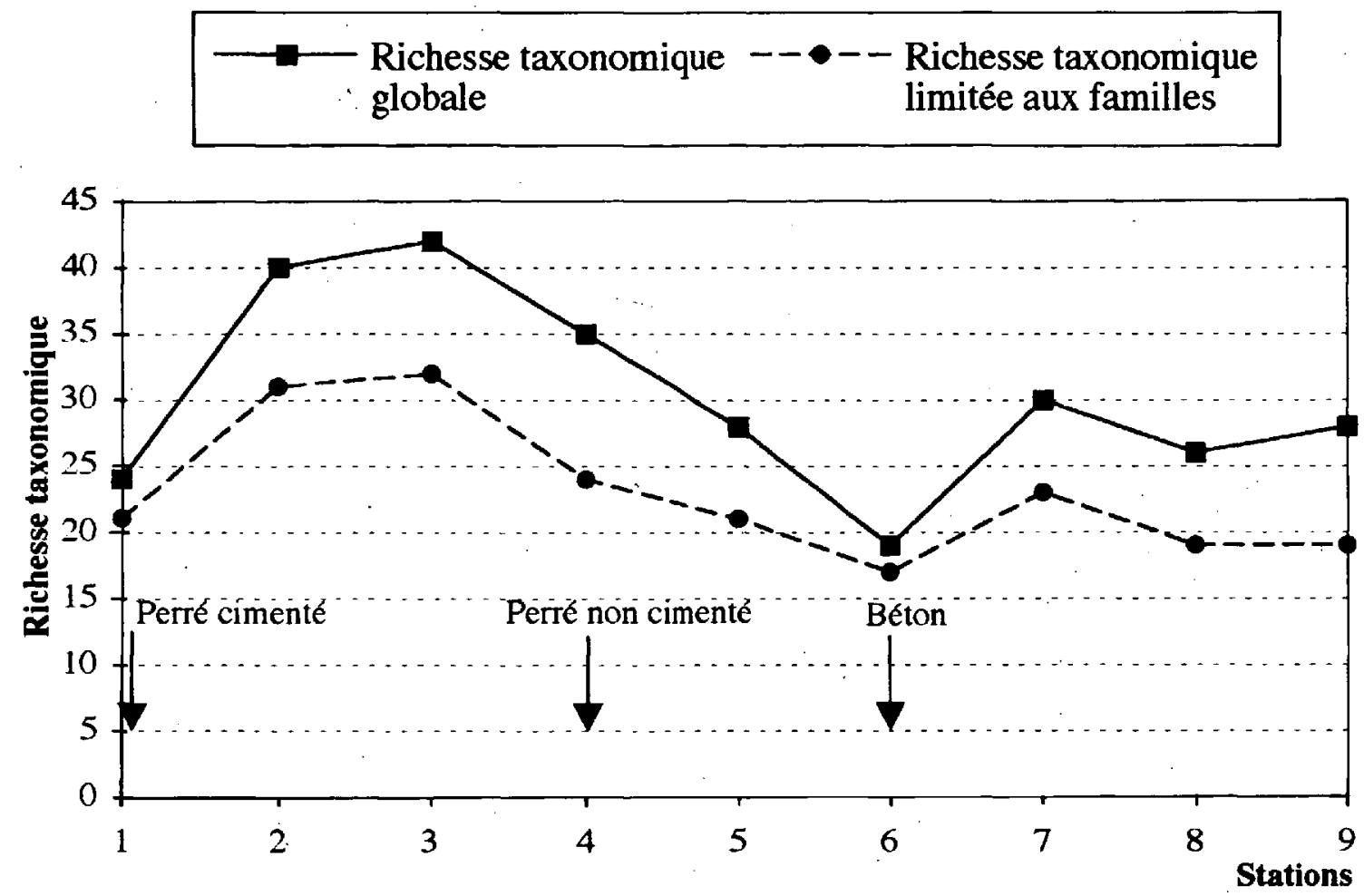

Fig. 5. Evolution du nombre de taxa, considérés à des niveaux de détermination différents et en fonction des substrats des 9 stations du bief de Waulsort (septembre 1989).

Fig. 5. Evolution of the taxa number following differents levels of identification and following substrates in 9 stations of the Waulsort reach (September 1989).

\subsection{Répartition des principales familles}

Le site pour lequel la colonisation par les Planariidae (Fig. 6) est la plus forte est la plage des galets de la station 2, que ce soit en nombre d'individus récoltés (2 116 individus) ou en abondance relative avec $15 \%$ de Dugesia lugubris. Les stations 3 et 4 avec leur substrat graveleux présentent une diversité identique de Planariidae, tout en comptant respectivement $7 \%$ et $4,5 \%$ de la population. Les Lumbriculidae nombreux aux stations $9(50 \%)$ et $5(33 \%)$ reflètent le substrat vaseux, propice pour ces limnivores. Quelques stations sont peu colonisées, à cause de l'absence de zone profonde à substrat meuble : le perré recouvert de béton $(2,5 \%)$ et le mur cimenté $(0,5 \%)$. La plage naturelle vaseuse de la station 5 présente une faune comprenant $13 \%$ de Sphaeriidae dont Psidium spp. La station 8, de substrat similaire, montre une proportion relativement élevée. Les substrats artificiels semblent inadéquats pour cette famille. Les quatres premières stations aux substrats érodés sont occupées par une proportion importante de Crustacés Corophiidae avec des variations de 2000 à 4000 individus selon la station. C'est surtout Corophium curvispinum qui abonde au dépens des Gammaridae. Dans l'ensemble des stations, on rencontre des proportions variables de Diptères Chironomidae. Ces proportions ont tendance à comprendre des représentants d'un genre distinct en fonction de la texture du substrat. Les galets et graviers de la station 2 sont principalement colonisés par Glyptotendipes spp. alors que le substrat caillouteux envasé de la station 7 est envahi par ce même genre accompagné de Chironomus spp. qui peuvent coloniser des substrats vaseux des grands fleuves eutrophes. 


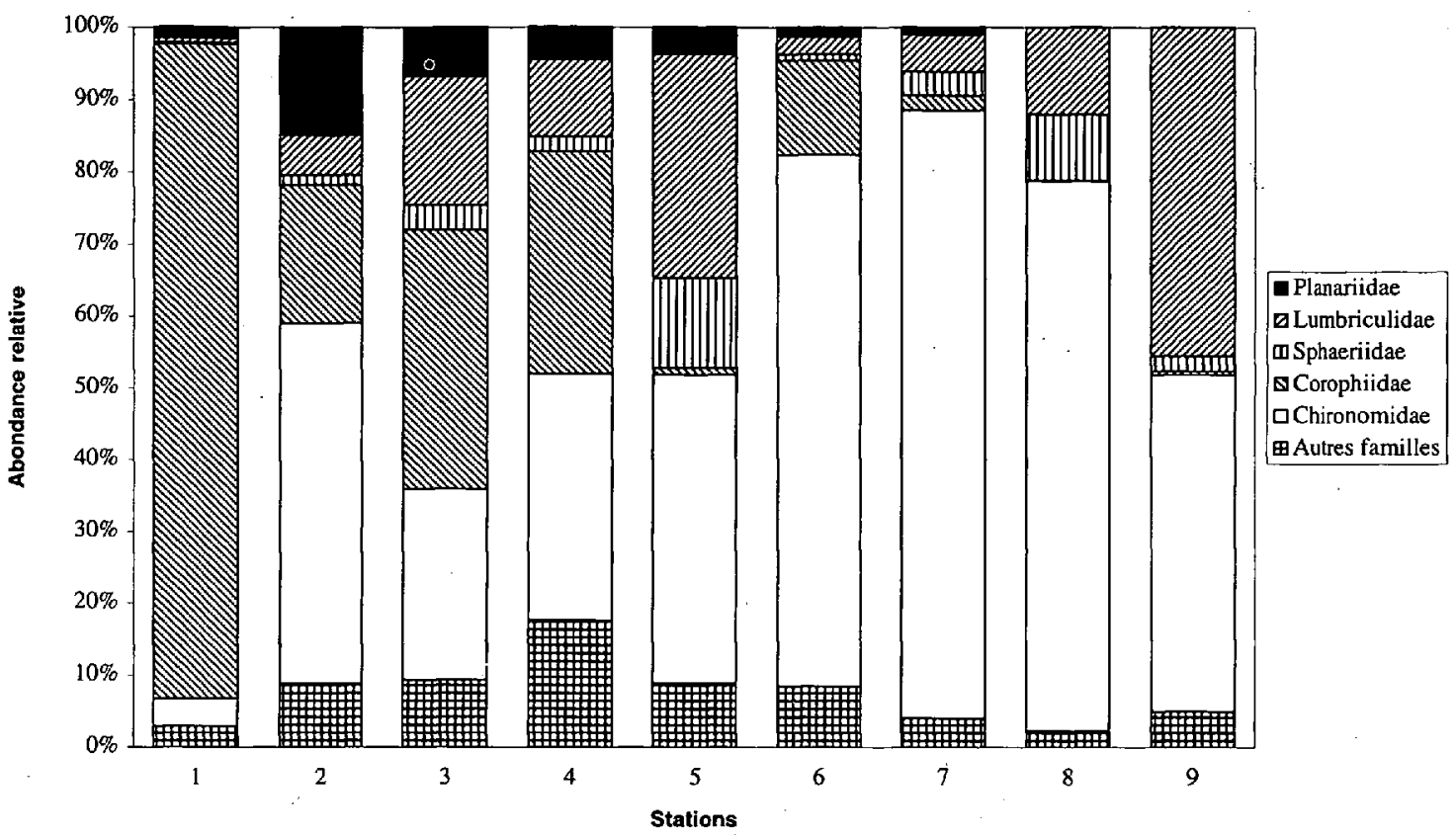

Fig. 6. Proportions des principales familles d'invertébrés benthiques dans les 9 stations du bief de Waulsort (septembre 1989). Fig. 6. Proportions of the main benthic invertebrates families in 9 stations of the Waulsort reach (September 1989).

L'effet de la proximité du barrage, mesuré à travers les valeurs de vitesse du courant, influence la faune de certaines stations. C'est ainsi que la station 2, directement située en aval du barrage de Waulsort, est composée de familles typiquement réhophiles avec la présence d'un Heptagenaeidae : Ecdyonurus sp. d'Athericidae, de quelques Trichoptères Hydropsychidae et Leptoceridae et de Mollusques Neritidae: Theodoxus fluviatilis ainsi qu'Ancylus fluviatilis. La première station, située dans les remous du barrage, présente également une faune rhéophile avec les Hydropsychidae et les Athericidae et Ecdyonurus sp. Au fur et à mesure de l'éloignement du barrage vers l'aval, l'ensemble du benthos retrouve les caractéristiques d'une faune limnophile avec les Lumbriculidae, les Sphaeridae sans compter les Chironomidae (Chironomus spp.) (stations 7, 8 et 9).

\section{Discussion}

L'objectif de notre travail était de déterminer la structure des communautés de macroinvertébrés benthiques ainsi que leur répartition sur différents substrats artificiels et naturels dans un bief de la Haute-Meuse belge (Waulsort). Les échantillonnages, réalisés à l'aide de quadrats et de filets Surber de même surface, ont montré des différences significatives de colonisation des substrats au sein du bief de Waulsort en fonction de leur texture, naturelle ou artificielle. La faune répertoriée reste typique de celle rencontrée dans les grands fleuves européens eutrophisés (Bournaud et al. 1980, Meurisse-Genin et al. 1987).

L'écosystème fluvial mosan est composé d'une mosaïque de microhabitats issus de l'action d'une multitude de facteurs biotiques et abiotiques qui en régissent les caractéristiques (Vannote et al. 1980, Bournaud 1983). La complexité de cet écosystème est responsable de la distribution des espèces en fonction de leurs exigences écologiques. La difficulté de caractériser le milieu en utilisant le substrat comme seul facteur influant, réside dans cette complexité qui se traduit par une importante variabilité des données. Cette variabilité de l'abondance ainsi que de la diversité reste marquée au niveau des stations à substrats pourtant similaires qui présentent des profils différents en fonction de la pente de la 
berge (stations 5.7 et 8 ), en fonction de la situation par rapport aux barrages (stations 2,3 et 4). La sélection des caractéristiques des stations, limitées à certains facteurs structurants supposés importants, comme la texture des différents substrats, a permis d'apporter un supplément d'informations dans l'explication des résultats apparemment typiques (Bournaud et al. 1980). Des études de densité faunistique sur des substrats caillouteux (Munoz \& Prat 1994) à des périodes comparables de l'année répertorient des densités similaires à celles rencontrées dans le bief de Waulsort, toutefois certaines ont été réalisées dans des cours d'eau moins importants (Minshall \& Minshall 1977, Reice 1980). La composition faunistique rencontrée près du barrage est typique de celle du potamon profond de rivières canalisées avec la présence d'espèces filtreuses et détritivores comme les Hydropsychidae associées avec des prédateurs essentiellement Hirudinées Erpobdellidae et Glossiphoniidae (Wiggins \& Mackay 1978).

Comme le souligne Bournaud (1983), si le substrat représente un facteur important qui influence considérablement la microdistribution des communautés de macroinvertébrés benthiques, d'autres facteurs abiotiques et biotiques la déterminent également (Lavandier \& Dumas 1971). La quantité de nourriture offerte aux espèces, la disponibilité en oxygène dissous, la vitesse du courant sont autant de facteurs biotiques et abiotiques qui conditionnent directement ou indirectement la répartition des espèces au sein des communautés benthiques. Ces relations multiples ne permettent pas toujours une compréhension totale des processus déterminant la microdistribution de ces communautés au sein de l'écosystème fluvial.

Dans le milieu étudié, les communautés benthiques présentent une caractéristique primordiale qui est leur diversité (Hynes 1970). Les substrats des berges de la Meuse dans le bief de Waulsort sont apparus comme des supports hétérogènes, ce qui n'est pas sans effet sur les communautés benthiques qu'ils abritent. Les variations de structure de ces communautés se font suivant la nature du substrat et suivant l'éloignement par rapport au barrage de Waulsort. D'après Verniers (1991), la comparaison du bief de Waulsort avec la plupart des autres biefs de la Meuse belge, essentiellement ceux qui ont été aménagés pour la navigation en Meuse moyenne et inférieure, montre les cas extrêmes de la recolonisation des berges par les macroinvertébrés benthiques
(Micha 1970, Meurisse-Genin et al. 1987). En effet, les berges artificielles offrent peu de possibilités de recolónisation, surtout lorsque l'homogénéité du substrat est parfaite. Cette étude fait ressortir des conclusions similaires, les substrats artificiels des perrés cimentés ou recouverts d'une coulée de béton sont beaucoup moins colonisés par la faune benthique. Dans la perspective de l'aménagement futur des berges du barrage de Waulsort, la construction de perrés non cimentés à faible pente doit être privilégiée.

L'approche quantitative de la relation faunemicrohabitat ou substrat est bien adaptée pour évaluer les effets des aménagements et pour préciser les causes réelles des modifications constatées dans les communautés benthiques. D'une manière plus générale, cette approche appliquée à des stations de la Meuse moyenne supérieure est susceptible de fournir des éléments de base pour une typologie quantitative de l'écosystème mosan qui servirait à apprécier la valeur écologique des diverses situations rencontrées.

A travers cette valeur de l'habitat, l'impact d'un aménagement du cours d'eau est mis en évidence. Les effets des aménagements sur les communautés de macroinvertébrés benthiques sont en général très marqués et conduisent à une réduction des potentialités de l'écosystème aquatique (Bournaud \& Cogerino 1986). D'un point de vue qualitatif, la faune présente une diversité d'autant plus élevée que le substrat est hétérogène, c'est le cas des substrats caillouteux et ceux constitués de galets et de graviers. Les paramètres quantitatifs de ces peuplements fournissent également des indications sur l'intensité de l'impact. Par le biais de cette étude, on pourrait imaginer, puisque la richesse et la diversité faunistiques renseignent sur la qualité du microhabitat autrement dit du substrat, que le substrat lui-même ainsi que les caractéristiques physiques majeures, puissent remplacer la communauté benthique afin de définir une valeur de l'habitat.

\section{Remerciements}

Nous remercions G. Verniers et A. Evrard pour leur aide technique.

\section{Travaux cités}

Bournaud M. 1983. - Le courant, facteur écologique et éthologique de la vie aquatique. Hydrobiologia, $21: 125-165$. 
Bournaud M. \& Cogerino L., 1986. - Les microhabitats aquatiques des rives d'un grand cours d'eau : approche faunistique. Annls Limnol, 22 : 285-294.

Bournaud M., Keck G. \& Richoux P. 1980, - Les prélèvements de macroinvertébrés benthiques en tant que révélateurs de la physionomie d'une rivière: Annls Limnol., 16 : 56-75.

Dajoz, 1971. - Précis d'écologie. Dunod, Paris : 433 p.

Hynes, 1970. - The ecology of running waters. Liverpool University Press : $555 \mathrm{p}$.

Lavandier P.\& Dumas P. 1971. - Microrépartition de quelques espèces d'invertébrés benthiques dans les ruisseaux des Pyrénées centrales. Annls Limnol., 7 : 7-23.

Meurisse-Genin M., Reydams-Detollenaere A., Stroot Ph. \& Micha J.-C. 1987. - Les macroinvertébrés benthiques de la Meuse belge : bilan de cinq années de recherche (1980 \& 1984). Arch. Hydrobiol., 109 : 67-88.

Micha J.-C. 1970. - Etude quantitative du benthos d'une rivière de Belgique : l'Ourthe liégeoise. Annls Limnol., 6 : 255-280.

Micha J.-C. \& Borlée M.-C. 1989. - Recent Historical Changes on the Belgian Meuse Historical Change of Large Alluvial Rivers : Western Europe. John \& Son, 16 : 269-295.

Micha J.-C. \& Pilette S. 1988. - L'impact de l'homme sur l'écosystème Meuse. Actes du colloque tenu à Namur (Belgique) les 3 et 4 novembre 1987. Presses Universitaires de Namur, 140 p.
Minshall G.W. \& Minshall J.N. 1977. - Microdistribution of benthic invertebrates in a Rocky Mountain (USA) stream. Hydrobiologia, 55 : 231-249.

Munoz \& Prat N. 1994. - Macroinvertebrate community in the lower Ebro river (NE Spain). Hydrobiologia, 286 : 65-78.

Reice S.R. 1980. - The role of substratum in benthic macroinvertebrate microdistribution and litter descomposition in a woodland stream. Ecology, 61 : 580-590.

Vannote R.L., Minshall G.W., Cummins K.M., Sedell J.R. \& Cushing C.E. 1980 . - The river continuum concept. Can. J. Fish. aquat. Sci., $37: 130-137$.

Verniers G. 1990. - Comparaison des différentes techniques de consolidation des berges. In Actes du Colloque « Gérer la nature ? ". Tra. cons. de la Nat., 15 : 703-710.

Verniers G., Gillet A., Micha J.-C., Rothilde C., Schiepper N. \& Paquet C. 1991. - Impacts écologiques des aménagements des cours d'eau navigables de Wallonie. Bulletin de l'Association Permanente des Congrès de Navigation, 73 : 27-37.

Wiggins G.B. \& Mackay R.J. 1978. - Some relationship between systematics and trophic ecology in neartic aquatic insects, with special references to Trichoptera. Ecology, 59 : 1211-1220. 